\title{
New localities of endangered Chinese turtles from museum specimens and the practical and ethical challenges using and reporting natural history collection data
}

\author{
JONATHAN J. FONG ${ }^{1} \&$ GE-XIA QIAO ${ }^{2,3}$ \\ ${ }^{\prime}$ Museum of Vertebrate Zoology; University of California, Berkeley; Berkeley, CA 94720; USA. E-mail: j_fong@berkeley.edu \\ ${ }^{2}$ National Zoological Museum of China, Institute of Zoology; Chinese Academy of Sciences, Chaoyang, Beijing 100101; China. \\ E-mail: qiaogx@ioz.ac.cn \\ ${ }^{3}$ Corresponding author
}

The turtle fauna of Asia is one of the most diverse in terms of number of species, but is also the most endangered. This is a result of human activities such as habitat destruction and over-harvesting for the pet, food, and medicine trades (van Dijk et al., 2000). Effective conservation measures require adequate baseline data on the ecology, systematics, and geographic distribution of each species. Unfortunately, Asian turtle research is difficult because of the longstanding, high-volume Asian turtle trade that includes long-distance transport. Not only are wild turtles increasingly difficult to find, those found in the field may have escaped or been released into the wild. In particular, because of the turtle trade, reconstructing the natural distributions of turtles becomes a problem; turtle records from Asia need to be treated with caution. A specific example of this situation was pointed out by Parham \& Li (1999), where some records from the pet trade proved to be misleading.

Currently, Iverson (1992) and "The EMYSystem" (Iverson et al., 2000) are the main resources available for geographical distributions of turtles. These resources include most published records for Asian turtles, but the distributions for several species are relatively unknown, being represented by only a handful of point localities. With new field records and by sifting through Chinese museums and Chinese literature, researchers have begun to improve these distributions (Parham \& Wang, 2000; Fong et al., 2002). Lessons learned from these studies are 1) current distributional data must be scrutinized due to the turtle trade, and 2) Chinese museums and literature hold valuable historical data, since many of the specimens pre-date the mass transport of turtles $(\sim 1980)$.

Access to data in Chinese museums is often difficult, due to the distance (interested people must travel to the museums, since catalogues are not electronically available) and language barriers (almost all of the records are in Chinese). However, these museums house valuable and often rare specimens and their associated data that can be used for research. Herein we translate the specimen catalogue for turtles in the Institute of Zoology, Chinese Academy of Sciences (IOZ). We outline a set of explicit criteria to evaluate the reliability of each specimen record, and provide updated distribution maps for the species where there are new data. In addition, we discuss two important issues that field researchers face when documenting locality data: collecting detailed locality data and the dangers of published data leading to the exploitation of the organism (dual-use dilemma).

A total of 12 pages of the IOZ catalogue, corresponding to the complete turtle collection, was translated and is presented in Appendix A. The appendix consists of verbatim Chinese data with English translations in parentheses. We have included three slight modifications to the catalogue. First, Chinese characters are converted from their original form in simplified characters to traditional characters. Next, common names, instead of being directly translated, follow the names found in Shi et al. (2008). Lastly, since the taxonomy of some species has changed, scientific names follow the current nomenclature by the Turtle Taxonomy Working Group (2007). 